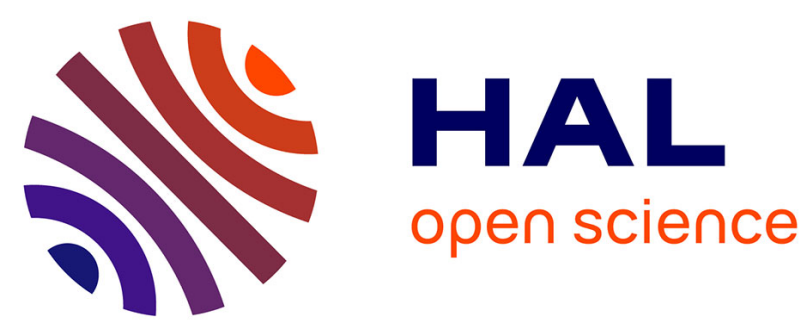

\title{
SDM Framework as a Support for Decision-Making Traceability in Design of Experiments Process
}

\author{
Farouk Belkadi, Luca Dall'olio, Gilles Besombes, Alain Bernard
}

\section{To cite this version:}

Farouk Belkadi, Luca Dall'olio, Gilles Besombes, Alain Bernard. SDM Framework as a Support for Decision-Making Traceability in Design of Experiments Process. 13th IFIP International Conference on Product Lifecycle Management (PLM), Jul 2016, Columbia, SC, United States. pp.275-285, 10.1007/978-3-319-54660-5_25. hal-01699691

\section{HAL Id: hal-01699691 \\ https://hal.inria.fr/hal-01699691}

Submitted on 2 Feb 2018

HAL is a multi-disciplinary open access archive for the deposit and dissemination of scientific research documents, whether they are published or not. The documents may come from teaching and research institutions in France or abroad, or from public or private research centers.
L'archive ouverte pluridisciplinaire HAL, est destinée au dépôt et à la diffusion de documents scientifiques de niveau recherche, publiés ou non, émanant des établissements d'enseignement et de recherche français ou étrangers, des laboratoires publics ou privés. 


\title{
SDM framework as a support for decision-making traceability in design of experiments process
}

\author{
Farouk Belkadi $^{1}$, Luca Dall'Olio ${ }^{2}$, Gilles Besombes ${ }^{3}$, Alain Bernard ${ }^{1}$ \\ Ecole Centrale de Nantes - IRCCyN - UMR CNRS 6597 \\ 1 rue de la Noë, Nantes 44300, France \\ ${ }^{2}$ ALNEOS - 196, rue Houdan, 92330 Sceaux, France \\ ${ }^{3}$ VALEO - Pôle systèmes thermiques centre R\&D La Verrière, France
}

\begin{abstract}
During the design of experiments process several simulations and experimentations are performed to evaluate all design solutions' alternatives. Technical meetings toke place regularly with the aim to select the product parameters and their values range to be considered in each simulation as well as the resolution methods and algorithms. One of the major problems concerns the lack of traceability and connections between all these design decisions and simulation results along the design process. A Simulation Data Management framework is proposed in this paper as a solution to resolve such kind of problems. This will enhance the final performance of the design of experiment process by reducing time of resolution and ensuring consistency of all simulation parameters, decisions and results.
\end{abstract}

Keywords: SDM; Design of Experiments, traceability,

\section{Introduction}

Nowadays, products are more and more complex composed by huge quantity of components and embedding large variety of technologies. The development process of such products requires the interactions of many actors, working on different elements of the design project and focusing on the same sub-system with different points of view (mechanical, electrical, FEM, etc.).

Engineering activities take central place in the development process and has become more and more challenging, requiring different tools and methods. Numerical simulation is one of the important stages of the engineering process to validate design decisions and to assess product performance along each step of its lifecycle [1]. Designs of Experiments (DoE) methods are often used to monitor the simulation process. To shorten the simulation process, two approaches were identified: (1) the DoE process execution duration reduction and (2) the DoE preparation process shortening by managing and reusing simulation data.

During these processes, product parameters, relations between these parameters, business constraints and rules, are allocated to interdependent sub-systems and considered on several computing algorithms with different objectives. Some parameters' values are resulted from the combination of other parameters, inputs of these simulation algorithms. This complexity requires one or more collaboration 
processes in order to help designers and decision-makers to converge their partial results into final and consistent result [2].

Due to these reasons, keeping the history of the design activity presents a great interest for the design project team and contributes significantly to shorten the process time and optimize the quality of the results. Traceability is usually associated with the activity of capturing, structuring and exploiting all information about the product, its parameters, states' evolutions, decisions and activities around this product, connected resources, tools, events, and the organization of these elements during the project [3].

Traceability is not an easier task because it needs the implication of several actors who are source of heterogeneous knowledge, generally implicit and difficult to formalize. The second reason is that asking individuals to keep formal trace of their activities in addition to realize these activities implies a significant cognitive workload, which can be a brake to such initiative.

In this context, it would be useful if designers could be encouraged to use the collaborative tools, enhanced by innovative technologies to keep a systematic record of the details of their activities and their corresponding situation.

In this context, one of the main challenges of the Simulation Data Management for Design of Experiment (SDM4DOE) project is to develop open-source simulation platform dedicated to DoE applications. This platform aims to support faster and efficient DoE processes, for complex numerical models, while ensuring traceability of generated data and providing a decision-aid system for DoE preparation.

This paper introduces concepts and specification to manage DoE process data. The DoE process characteristics are firstly described to highlight the need of traceability and data management in such complex process. The DoE data models developed to solve this issue is presented in the third section. The last section gives an overview of the SDM platform focusing on the traceability and data management functions.

\section{Data management and Traceability in DoE Process}

\subsection{Design of Experiment Process}

A DoE is a set of experiments defined to assess the numerical model for different configurations of the product. A DoE is defined by its type (distribution of experiments in the design-space), the number and type of its factors (model parameters) and associated levels. A DoE process may have different objectives (exploration, product optimization, sensitivity analysis, etc.) and submitted to different constraints (e.g. computational budget). The computational cost of a DoE is the cost of the numerical model calculation multiplied by the number of experiments. Thus, an optimal strategy is to choose the most efficient DoE and to use a method for reducing the computational cost of each run.

The DoE process is based on the simulation process, which consists of three main steps [4] (Figure 1). First, a parameterized numerical model of the studied system is created. Corresponding outputs (design parameters) are obtained by a specific solver and analyzed during the post-processing step, for model checking and product validation. 
After the definition and the validation of the numerical model [5], the available factors must be analyzed and selected to reduce the DoE cost, by a sensitivity analysis in the preparation step before starting simulations. Only most influent factors are kept, according to a specific output. To analyze accurately these influences, a meta-model (or surrogate model) is created in the last step from DoE results and statistical methods, as ANOVA. The meta-model can be reused to replace the numerical model for other studies in order to save time and cost.

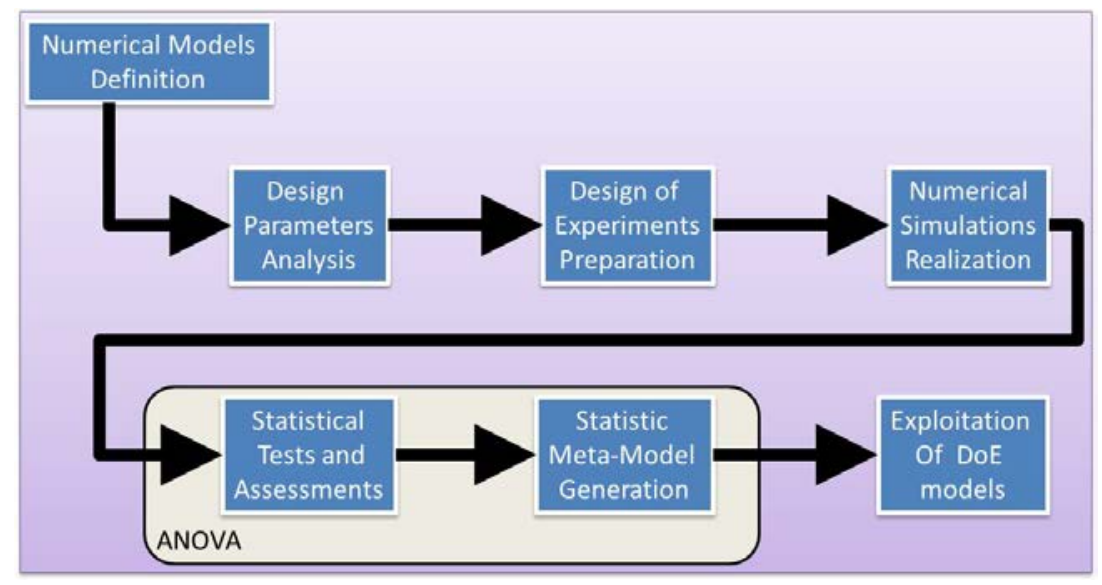

Fig 1. Design of experiment process

All of these steps may generate a large amount of heterogeneous data and could be very expensive and time-consuming. An efficient DoE should minimize the number of runs and optimize the space-covering of the runs, according to the DoE objective (exploration, product optimization...).

\subsection{Need of traceability}

Several types of DoE are available for numerical simulation [6] depending on its properties (space-filling, uniformity, etc.), objectives (exploration, optimization, etc.) and constraints (computational budget, output linearity properties, etc.). DoE type selection can be a long and difficult operation [7], needing assistance through the classification of all DoE types, simulation methods and used meta-models. Metamodels are used to replace a costly numerical model by a function faster to be assessed, for a specific output. The complexity and variety of meta-models depend on the DoE type and the business domain [8]. Although large quantities of methods and meta-models exist and some classification initiatives are already started, the need of robust tools to assist the hard task of identification of the best DoE methods and metamodels still a critical aspect to improve the design process.

In addition, the DoE process is a collaborative process in which several experts from different fields with different roles are involved. The effect of DoE results on the global product development process implies many interactions between DoE Process 
and upstream and downstream activities. Many choices, tests and decisions are made as a consequence of several iterations that take place on separate working sessions. Stakeholders working on a new session need to be aware about all decisions and choices generated in previous sessions, especially if they are made by other experts.

Thus, a DoE run involves a large amount of heterogeneous data. Some of these data needs to be standardized and classified to ensure easier knowledge finding and reuse on one hand, and a good communication between involved stakeholders as well used business tools, in the other hand. Implementation of Data modeling strategy may lead to reuse data and help the designer to avoid missing and errors.

\section{Data models for DoE traceability}

Simulation Data Management (SDM) [9] is a part of a larger issue of Product Data Management (PDM). PDM provides methods and tools to support the structuring, storage in shared repositories, management and sharing of product-related data and processes for its processing. More recently, SDM issues are associated with the new Product Lifecycle Management approaches (PLM) [10]. This covers technical data management systems of the product (PDM), the design support tools (CAD/CAE), manufacturing support tools (CAM) and other ERP applications [11]. Numerical DoE is a concrete example of the use cases of SDM tools. The realization of a DoE is based on the combined use of a set of design tools, simulation, computation, statistical processing and control of the computing process.

\subsection{DoE concepts classification}

In the context of the SDM4DOE project, a first study was realized to map and classify all data involved in a DoE process. The global organization of main data types are classified in the following items:

- The package "Design of Experiments": this first category of data aims to describe the main properties of DoE: objectives, the type, the nature of the studied phenomenon, etc.

- The package "Traceability and Administration" is used to link the DoE folder with its administrative and operational environment. This connection is done by several concepts such as: the reference of the project, the product and / or component associated with the DoE, the stakeholders involved in the working sessions, the timing of these working sessions with related decisions, etc.

- The package "Parameters" is the central node of the DoE data model. Through this concept the different types of parameters (factors) are classified according to their nature, their input/output status in the different steps of the DoE process and the possible intervals of variation of their value. The concept of "parameter" is used to define other specific properties but also to provide a common semantic for the codification of parameters' name. These concepts will allow communication between involved enterprise business platforms and with the SDM platform. 
- The package "Business Models" is used to manage in a uniform way all types and versions of business models involved in a DoE process to facilitate their sharing. It also links parameters to business models. It mainly involves CAD models, FEM and meta-model data.

- The package "Storage-Representation" provides important information to SDM for data location identification for extraction and exploitation. Because of their heterogeneity and diversity of their sources, data are stored in different places and in different formats (databases, header files, etc.) according to their nature.

- The package "Simulation-Computing" lists all types of simulation scripts and possible methods of analysis in a DoE process according to a given problem in a specific domain. It also links these types of processing with concerned parameters.

- The package «Resources» completes the definition of processing by proposing a classification of all software and methodologies supporting the computations. This will allow SDM users to quickly define the various steps of the DoE. The concept of "computational cost" allows a better characterization of the various alternatives of computations to facilitate the selection of the DoE execution mode.

\subsection{Traceability of Administrative data}

The traceability of administrative and decision making justification is made around the concept of DoE folder. Figure 2 illustrates the main concepts required for the representation of all administrative data for traceability issues. The concept of "DoE folder" is the classifier of all DoE realizations according to one alternative solution of the product (or component) and one physical analysis. Each DoE realization consists on the concrete selection of parameters and their value intervals, but also the execution of all simulations required by the selected physical analysis.

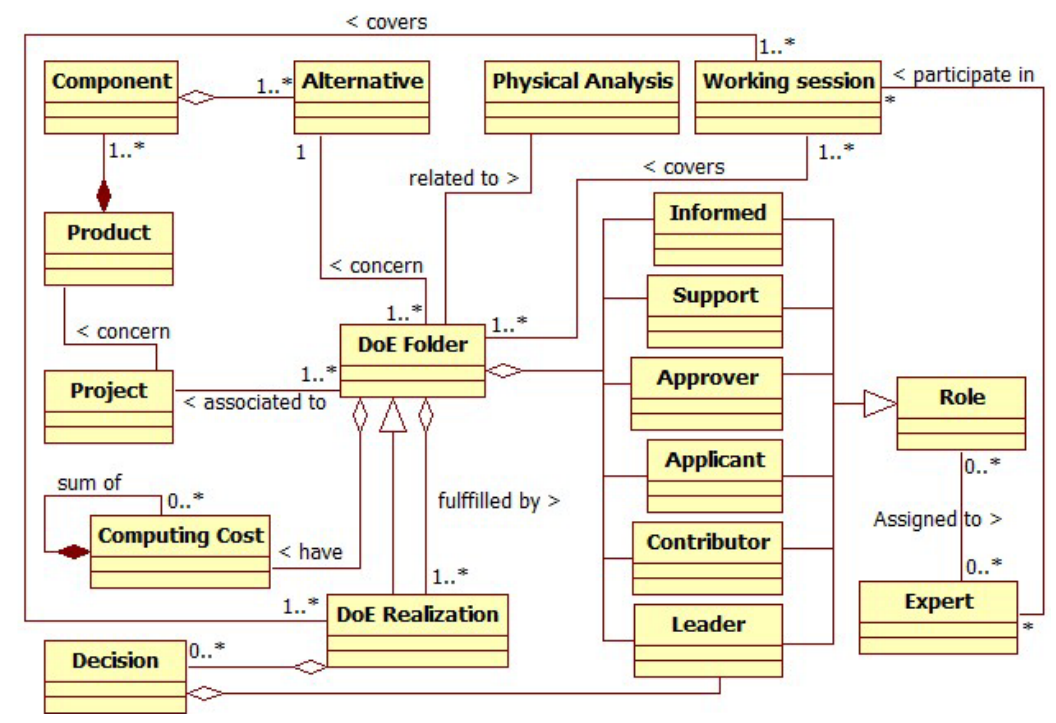

Fig 2. The DoE Folder 
The DoE folder is associated to a specific project and it is the space of interaction of several experts. Each expert takes one or more roles in the DoE process. These roles can be changed during the real physical meetings. These meetings are represented by the class "working session", in which one or more DoE Folders and/or realization might be occurred.

For traceability and future reuse perspective, the different decisions taken during concrete realization are stored in the related class. The total computing costs of different realizations is described in the DoE folder as an indicator for future choice of simulation methods and scripts in similar situations.

\subsection{Traceability of technical data of the DoE}

The main stage of DoE folder definition consists on the selection of parameters from the business model (FEM file). Then, the concrete realization of the DoE will start by the definition of variables inputs as instances of a sub set of the selected parameter (Figure 3). The value variation of these instances could be obtained through fixed number, a mathematical formula or other random sampling generated by the Sampler module of Uranie software. The DoE realization result is obtained as a set of instances after a set of simulations and mathematical treatments (optimization, Anova, etc.).

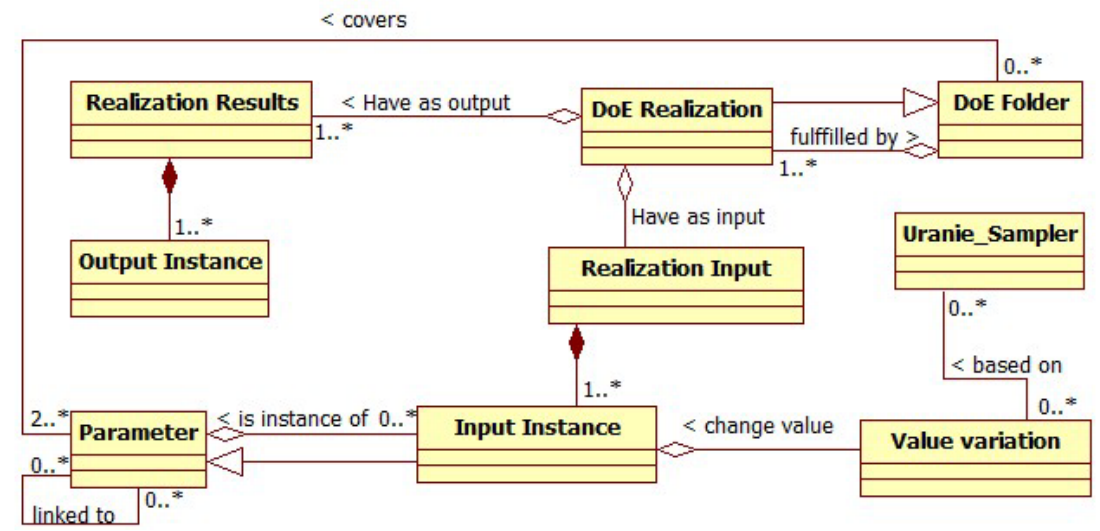

Fig 3. Traceability of technical data

\section{Models Implementation in the SDM framework}

To implement a DoE data management system, open-source software architecture is being developed in the SDM4DOE project. This architecture is developed as a SaaS web service, based on several open-source solutions. This section gives an overview of the developed platform focusing on the main data management functionalities. 


\subsection{Functional architecture}

As it is shown in the Figure 4, the SDM platform supporting collaborative DoE process provides users a set of processing rules and management facilities of administrative and technical data during a work session. This is fulfilled via a webbased interface connected at to a cloud solution and provided from a web-server. It also allows viewing and navigating the previous PEN traceability data. To achieve processing features, the Uranie open-source software integration is launched in a computing cluster at the end-back layer. The kernel of the SDM framework is the shared workspace which makes connection between the web server and the company' computing cluster and includes several functionalities of data sharing and reporting.

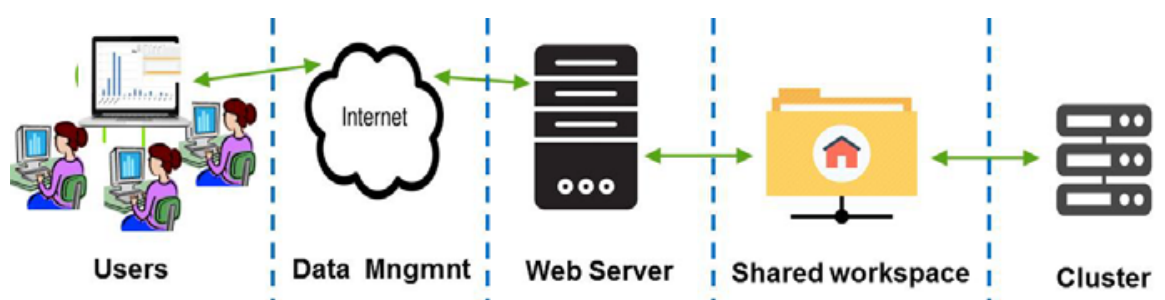

Fig 4. Global architecture of the SDM Framework

The computation chain of a DoE in the company's computing cluster is supervised by post-processing software Salomé. These services include the simulation software Code_Aster, in which acceleration methods of computations will be implemented.

Several functionalities are offered by the SDM framework to support the DoE process. The main interactions to fulfil these functionalities are summarized in the UML use case diagram bellow (Figure 5). According to this diagram, the user can create new DoE folder and several realizations of this folder. At any moment he can edit the properties of an existing DoE folder and create new folder as an adaptation of existing one. This will help time saving and data messing especially when creating new version or variant of existing products (i.e. component).

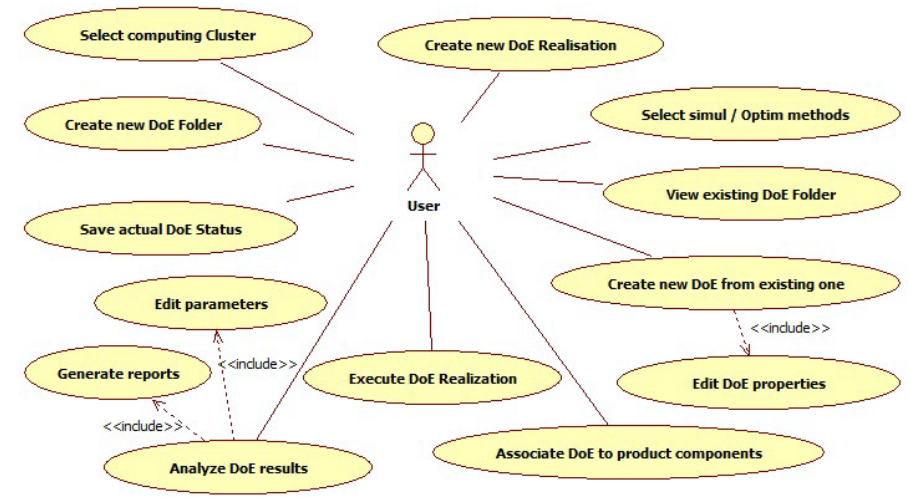

Fig 5. Main interactions with the SDM framework 
Finding and Reuse functionalities are particularly appreciate when the user is searching for the optimal list of parameters and simulation method he should consider for his current problem. Knowledge base repository is used to support this functionality [12]. To perform a DoE realization, the connection of the workspace to different computing clusters allows users to verify availability and status of different cluster in order to select the most suitable regarding to the complexity of the planned simulations. To analyze the results, two possibilities are offered: generating different reports with predefined set of information, and dynamic interface on which the user can observe the output of the meta-model for each combination of parameter values.

\subsection{SDM GUIs Overview}

To illustrate the principle of functioning, this section presents few Graphical User Interfaces used in the first steps of the DoE process. The starting point is already the creation of new working session (Figure 6) that groups heterogeneous experts to take decisions about the current DoE. This will prepare the DoE execution by the identification of the available clusters and the clarification of the DoE objectives, the role of each participant and the concerned product components.

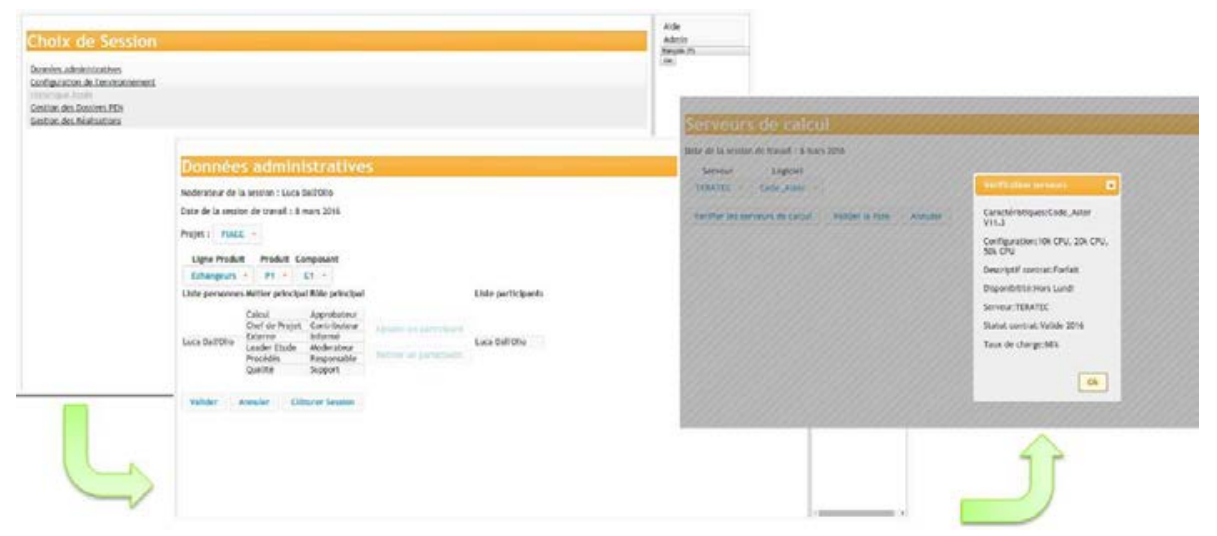

Fig 6. SDM GUIs : New DoE working session

After fixing the administrative data, useful to handle decisions and results traceability, the user can decide to create a new DoE Folder, open existing one, create new DoE realization or view the results of last one and send execution order to the selected cluster. The manipulation of DoE folders and realizations implies the manipulation of related parameters, displayed in a separate window with default values (Figure 7). 


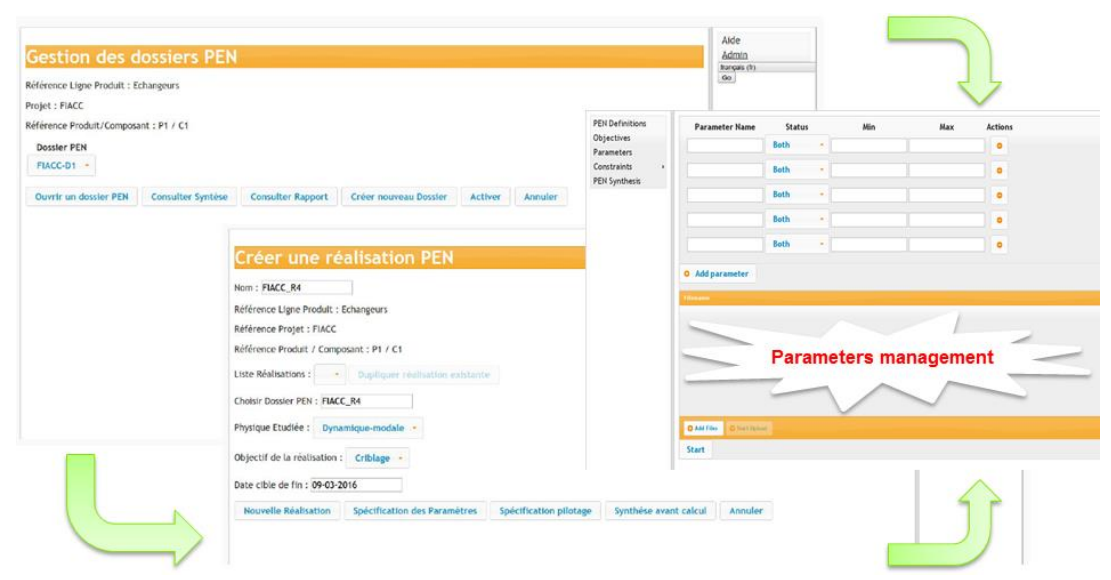

Fig 7. SDM GUIs : DoE folder and instance

At any time, the user can edit the synthesis of the current DoE folder, all related realization as well the status of all launched simulations. Automatic workflow notifies the end of simulation. Then the user can request the results, which he can view and navigate on a separate window (Figure 8). If the result is validated, it can be stored to be reused in future projects.

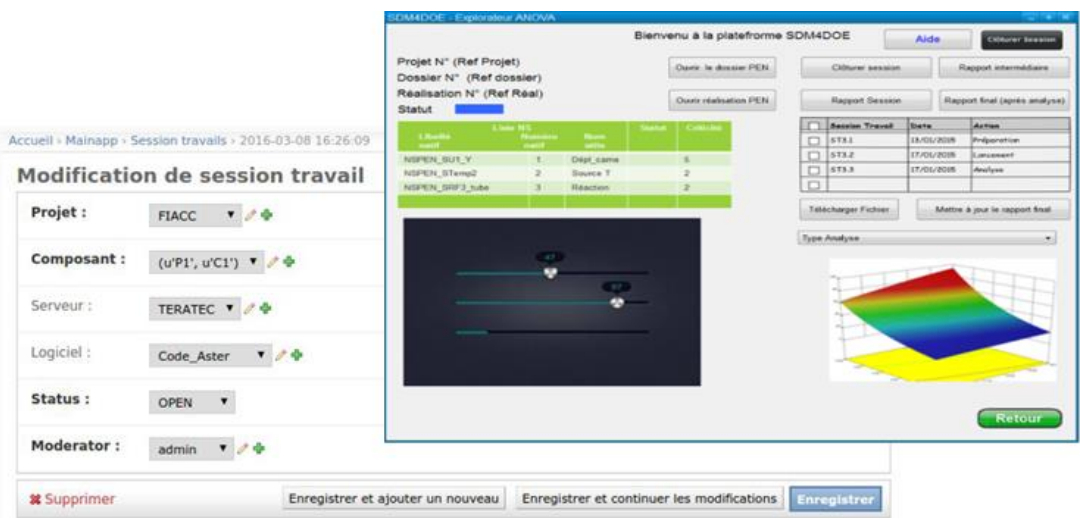

Fig 8. SDM GUIs : Edition of DoE synthesis and results

\section{Conclusion}

The first results of the SDM4DOE project show that existing SDM tools are not adapted to the heterogeneous nature of the DoE process data. Indeed, the optimization of a DoE process is based on the effective management of technical data, administrative data and also data related to the traceability of decisions. At this moment, a set of concepts has been proposed and is being refined and validate. 
The next step consists on testing the proposed concepts through the application of the first version of the SDM platform in realistic industrial use cases. On the other hand, the mastery of the DoE complexity will be based on a standard codification proposal, on both business files and data types, to ensure the integration of SDM4DOE solution for all types of business computing platforms.

Acknowledgments. This work is funded by the French FUI project SDM4DOE and labeled by French competitiveness clusters SYSTEMATIC and ID4CAR. We also thank all consortium partners for their contribution during the development of ideas and concepts proposed in this paper. http://www.id4car.org/SDM4DOE.html

\section{References}

1. Bernard, A, Chenouard R (2014) Multi-physics simulation for product-service performance assessment. In: 6th CIRP Conference on Industrial Product-Service Systems, vol. 16, Windsor, Ontario, Canada, p. 21-5. http://dx.doi.org/10.1016/j.procir.2014.03.002.

2. Kleiner S, Anderl R, Gräb R (2003) A collaborative design system for product data integration. Journal of Engineering Design, 14(4): 421-428.

3. Brand S.C (2005) A Process Data Warehouse for Tracing and Reuse of Engineering Design process. In: The Second International Conference On Innovations in Information Technology ICIIT’05, Dubaï

4. Charles S (2005) Gestion intégrée de données CAO et EF. Contribution à la liaison entre conception mécanique et calcul de structures, Ph.D. Thesis, Univ. Technologie de Troyes.

5. Giles Jr. H.F, Wagner Jr. J.R, Eldridge M. M.I (2014) Design of Experiments, Extrusion: The Definitive Processing Guide and Handbook Plastics Design Library, 291-308.

6. Chen V.C.P, Tsui K.L, Barton R.R, Meckesheimer M (2006) A review on design, modeling and applications of computer experiments. IIE Transactions, 38(4): 273-91.

7. Simpson T.W, Peplinski J.D, Koch P.N, Allen J.K (2001) Metamodels for computer-based engineering design: survey and recommendations. Engineering with Computers, 17(2): 129-50.

8. Castric S, Denis-Vidal L, Cherfi Z, Blanchard G.J, Boudaoud N (2012) Modeling Pollutant Emissions of Diesel Engine based on Kriging Models: a Comparison between Geostatistic and Gaussian Process Approach.14th IFAC Symposium on Information Control Problems in Manufacturing, INCOM'12, Bucharest, vol. 14: 1708-15.

9. Charles S, Ducellier G, Eynard B (2006) CAD and FEA integration in a simulation Data Management environment based on a knowledge based system. TMCE, Ljubljana: 1719-30

10. Assouroko I, Ducellier G, Belkadi F, Eynard B, Boutinaud P (2010) Improvement of engineering design and numerical simulation data exchange based on requirements deployment: a conceptual framework. 7th International Conference on Product Lifecycle Management, Bremen, Germany, pp 487-97.

11. Bosch-Mauchand M, Belkadi F, Bricogne M, Eynard B (2013) Knowledge based assessment of manufacturing process performance: integration of product lifecycle management and value chain simulation approaches. International Journal of Computer Integrated Manufacturing, 26(5): 453-473.

12. Blondet G, Belkadi F, Le Duigou J, Boudaoud N. (2016) Towards a knowledge based framework for numerical design of experiment optimization and management. ComputerAided Design and Applications, vol. 13(6): 872-884. 